of MTX withdrawal in patients achieving good clinical response to TCZ+MTX (COMBO) has not been evaluated.

Objectives: To evaluate whether TCZ-MONO is non-inferior to TCZ-COMBO in maintaining clinical response in patients who achieve low disease activity with TCZ-COMBO.

Methods: US patients with RA who were inadequate responders to MTX were enrolled: initial combination therapy included MTX (15 mg/week orally) plus TCZ $162 \mathrm{mg}$ subcutaneous (SC) either weekly (qw; for patients $\geq 100 \mathrm{~kg}$ ) or every 2 weeks (q2w; for patients $<100 \mathrm{~kg}$ ). Dose escalation from q2w to qw was allowed at week 12 in patients who had not achieved low disease activity (DAS28 $\leq 3.2$. If patients achieved DAS28-ESR $\leq 3.2$ at week 24 , they were randomized (double-blind) 1:1 to receive either TCZ-MONO or continue TCZ-COMBO until week 52. Patients who did not achieve DAS28 $\leq 3.2$ were assigned to an open-label arm and continued TCZ-COMBO until week 52. The primary outcome measured was the comparison of mean change in DAS28-ESR score in the randomized cohort between weeks 24 and 40, between the TCZ-MONO or TCZ-COMBO arms (noninferiority margin of 0.6 ). Secondary outcomes included the proportion of patients achieving DAS28 $<2.6$, DAS28 $<3.2$ and American College of Rheumatology $20 \% / 50 \% / 70 \%$ (ACR20/50/70) responses at weeks 40 and 52, and safety. Trial registration number: NCT01855789.

Results: Of 718 patients enrolled, 296 were randomized at week 24 (TCZ-MONO, $n=148$; TCZ-COMBO, $n=148$ ). Early discontinuation in the randomized cohort occurred in $12.2 \%$ of patients in the TCZ-MONO group and $10.2 \%$ in the TCZ-COMBO group. Baseline characteristics were balanced between treatment groups (mean age 55.5 years; $74.8 \%$ female; mean RA duration 6.8 years; mean DAS28-ESR 6.3). At week 24, DAS28 scores were similar in both groups, but ACR responses were $\sim 8-11 \%$ lower in the TCZ-MONO group prior to MTX withdrawal (randomization). The mean change in DAS28 was similar between the randomized treatment groups (Table 1). For the primary efficacy analysis, the mean changes in DAS28 from week 24 to week 40 were 0.46 and 0.14 in the TCZ-MONO and TCZ-COMBO groups, respectively (95\% Cl: $0.045-0.592)$. This study met the primary endpoint by demonstrating that discontinuing MTX in TCZ responders was noninferior to continuing MTX. The safety of TCZ-SC in this study was consistent with the known safety profile with no new safety signals observed (Table 2). The most common SAE was infection, occurring in $4.1 \%$ of patients. TCZ-COMBO had greater frequency of AEs, SAEs and serious infections than TCZ-MONO.

\begin{tabular}{|c|c|c|c|c|}
\hline & $\begin{array}{c}\text { TCZ-MONO } \\
n=147\end{array}$ & $\begin{array}{c}\text { TCZ-COMBO } \\
n=147\end{array}$ & \multicolumn{2}{|c|}{$\begin{array}{c}\text { Difference }(95 \% \mathrm{Cl}) \\
\text { (TCZ-MONO minus } \\
\text { TCZ-COMBO) }\end{array}$} \\
\hline \multicolumn{5}{|c|}{$\triangle \mathrm{DAS} 28-\mathrm{ESR}$, Mean $(\mathrm{SEM})^{\star}$} \\
\hline Week 24 to week 40 & $0.46(0.123)$ & $0.14(0.126)$ & \multirow{2}{*}{\multicolumn{2}{|c|}{ 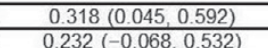 }} \\
\hline Week 24 to week 52 & $0.43(0.136)$ & $0.20(0.139)$ & & \\
\hline \multicolumn{5}{|c|}{ Response at Week $40, \mathrm{n}(\%)$} \\
\hline DAS28 $\leq 3.2$ & $94(63.9)$ & $113(76.9)$ & \multicolumn{2}{|c|}{$-12.9(-23.3,-2.6)$} \\
\hline DAS28 $<2.6$ & $74(50.3)$ & $87(59.2)$ & \multicolumn{2}{|c|}{$-8.8(-20.2,2.5)$} \\
\hline ACR20 & $103(70.1)$ & $116(78.9)$ & \multicolumn{2}{|c|}{$-8.8(-18.8,1.1)$} \\
\hline ACR50 & $76(51.7)$ & $94(63.9)$ & \multicolumn{2}{|c|}{$-12.2(-23.4,-1.0)$} \\
\hline ACR70 & $51(34.7)$ & $62(42.2)$ & \multicolumn{2}{|c|}{$-7.5(-18.6,3.6)$} \\
\hline \multicolumn{5}{|c|}{ DAS28 Worsening $\geq 1.2, \mathrm{n}(\%)$} \\
\hline Week 24 to week 40 & $42(28.6)$ & $31(21.1)$ & \multicolumn{2}{|c|}{$7.5(-2.4,17.3)$} \\
\hline \multicolumn{5}{|c|}{$\begin{array}{l}\text { ACR, American College of Rheumatology criteria; AE, adverse event; } \triangle \mathrm{DAS} 28 \text {-ESR, change in Disease } \\
\text { Activity Score-28 joints erythrocyte sedimentation rate; MTX, methotrexate; SAE, serious adverse } \\
\text { events; SEM, standard error of the mean; TCZ-COMBO, tocilizumab plus MTX; TCZ-MONO, TCZ } \\
\text { monotherapy. } \\
\text { *Adjusted means from ANCOVA model include week } 24 \text { DAS28 as a covariate, treatment group and the } \\
\text { randomization stratification factors: DAS2 } 28 \text { remission status at week } 24(<2.6 ; \geq 2.6 \text { to } \leq 3.2) \text {, patient } \\
\text { anti-TNF exposure (Yes/No), baseline weight-by-dosing group ( }<80 \mathrm{~kg} q 2 \mathrm{w} ;<80 \mathrm{~kg} \text { qw; } 80 \text { to }<100 \mathrm{~kg} \\
\text { q2w, } 80 \text { to }<100 \mathrm{~kg} q w, \geq 100 \mathrm{~kg} \text { qw). Last observation carried forward (LOCF) was used to impute } \\
\text { missing data at week } 40 \text { only. } \\
\text { Table 2. Safety of TCZ as Monotherapy and in Combination With MTX }\end{array}$} \\
\hline $\begin{array}{l}\text { Rate, per } 100 \mathrm{PY} \\
(95 \% \mathrm{Cl})\end{array}$ & $\begin{array}{c}\text { Total }^{*} \\
N=713 \\
700.60 \mathrm{PY}\end{array}$ & $\begin{array}{r}\text { TCZ-M } \\
n=1 \\
92.44\end{array}$ & $\begin{array}{l}\mathrm{NO}^{\dagger} \\
44 \\
\mathrm{PY}\end{array}$ & $\begin{array}{c}\text { TCZ-COMBO }^{\dagger} \\
n=139 \\
90.56 \mathrm{PY}\end{array}$ \\
\hline AEs & $\begin{array}{c}377.1 \\
(362.9,391.8\end{array}$ & $\begin{array}{r}238 \\
(207.6\end{array}$ & $271.6)$ & $\begin{array}{c}308.1 \\
(273.0,346.4)\end{array}$ \\
\hline SAES & $\begin{array}{c}17.0 \\
(14.1,20.3)\end{array}$ & $\begin{array}{r}8.7 \\
(3.7 .1\end{array}$ & & $\begin{array}{c}14.4 \\
(7.6,24.6)\end{array}$ \\
\hline Serious infections & $\begin{array}{c}5.0 \\
(3.5,7.0)\end{array}$ & $\begin{array}{r}3 . \\
(0.7 \\
\end{array}$ & & $\begin{array}{c}4.4 \\
(1.2,11.3)\end{array}$ \\
\hline
\end{tabular}

t Includes all randomized patients who received TCZ+MTX or TCZ+PBO from week 24 to end of study

Conclusions: These results demonstrate that patients receiving TCZ-COMBO who achieve low disease activity can discontinue MTX and maintain disease control.

Acknowledgements: This study was funded by F. Hoffmann-La Roche, Ltd. Disclosure of Interest: J. Kremer Shareholder of: Corrona, LLC, Consultant for: AbbVie; Amgen; Bristol-Meyers Squibb; Genentech; GlaxoSmithKline; Eli Lilly; Pfizer; Regeneron; Sanofi, Employee of: Corrona, LLC, W. Rigby Consultant for: Roche, N. Singer Grant/research support from: Merck, EMD Serono, Consultant for: Pfizer, C. Birchwood Employee of: Genentech, Inc., D. Gill Employee of: Genentech, Inc., W. Reiss Employee of: Genentech, Inc., J. Pei Employee of: Genentech, Inc., M. Michalska Employee of: Genentech, Inc.

DOI: 10.1136/annrheumdis-2017-eular.2797

\section{FRI0223 ANTI-CCP IS AN INDEPENDENT PREDICTOR OF 12-MONTH EULAR RESPONSE IN PATIENTS WITH RA TREATED WITH ABATACEPT}

A. den Broeder ${ }^{1}$, T. Kerstens ${ }^{2}$, J. Fransen ${ }^{2}$, C. van den Ende ${ }^{1}$,

L. Tweehuysen ${ }^{3}$, R. Postema ${ }^{4}$, E. Alemao ${ }^{5}$, F. van den Hoogen ${ }^{2} .{ }^{1}$ Sint Maartenskliniek and Radboud UMC; ${ }^{2}$ Radboud UMC; ${ }^{3}$ Sint Maartenskliniek, Nijmegen, Netherlands; ${ }^{4}$ Bristol-Myers Squibb, Uxbridge, United Kingdom;

${ }^{5}$ Bristol-Myers Squibb, Princeton, United States

Background: Although anti-cyclic citrullinated peptide (anti-CCP) positivity is regarded as a strong prognostic factor for untreated RA outcome, the benefit of anti-CCP tests for personalized medicine is controversial. ${ }^{1}$ Illustratively, anti-CCP was not predictive for response to anti-TNF in RA, as shown in meta-analyses, although some predictive value was shown for rituximab. ${ }^{2-4}$ There are, however, indications that better response to abatacept (ABA) is predicted by anti-CCP positivity. ${ }^{5-7}$

Objectives: To test whether anti-CCP level at baseline $(B L)$ is an independent predictor for treatment response (DAS28 [CRP]-based EULAR response criteria) at 12 months $(M)$ in patients (pts) with RA treated with $A B A$.

Methods: Consenting pts with RA from Radboud UMC and Sint Maartenskliniek were consecutively included if they started treatment with $A B A(B L)$. The anti-CCP values closest before BL were used. DAS28 (CRP) was assessed at BL and at $12 \mathrm{M}$ by trained rheumatology nurses or rheumatologists. Demographic and disease-related variables, treatment history and co-morbidity were also assessed. Primary outcome was response to treatment based on DAS28 (CRP) EULAR response criteria at M12. Therapy cessation was regarded as non-response. Multiple imputation with 20 repetitions was used to replace missing predictors. Multivariate logistic regression was used to examine whether anti-CCP positivity was an independent predictor for treatment response, taking confounding $\mathrm{BL}$ covariates (Table variables) into account.

Results: Data were available for 200 pts with RA starting ABA. Mean (SD) age was 58 (13) years, $165(83 \%)$ were female and median (p25-p75) disease duration was 12 (7-19) years (Table). Overall, $121(61 \%)$ pts were anti-CCP positive at BL. At 12M, 86 (43\%) pts remained on ABA. In the univariate model, anti-CCP was a predictor for treatment response (odds ratio $2.51 ; 95 \% \mathrm{Cl} 1.1$, $6.0 ; p=0.038$ ). No relevant confounding was present.

Table 1. Baseline Characteristics

\begin{tabular}{lc}
\hline & Abatacept $(\mathrm{n}=200)$ \\
\hline Age, years, mean (SD) & $58(13)$ \\
Female, $\mathrm{n}(\%)$ & $165(83)$ \\
$\mathrm{RF}+, \mathrm{n}(\%)$ & $128(64)$ \\
Anti-CCP+, $\mathrm{n}(\%)$ & $121(61)$ \\
No. of previous bDMARDs & $3(3-4)$ \\
No. of previous csDMARDs & $3(2-4)$ \\
Oral glucocorticoids, $\mathrm{n}(\%)$ & $79(40)$ \\
Disease duration, years & $12(7-19)$ \\
Treatment duration, months & $8(4-28)$ \\
NSAID, $\mathrm{n}(\%)$ & $117(59)$ \\
Concomitant DMARD, $\mathrm{n}(\%)$ & $117(59)$ \\
Overweight (BMI $\left.>25.0 \mathrm{~kg}^{\star} \mathrm{m}^{2}\right), \mathrm{n}(\%)$ & $98(48)$ \\
\hline
\end{tabular}

${ }^{*}$ Median (p25-p75). RF+: IgM-Rheumatoid factor positivity. b/csDMARD=biologic/conventional synthetic DMARD.

Conclusions: Anti-CCP positivity was confirmed as an independent predictor for treatment response at $12 \mathrm{M}$ in pts with RA treated with abatacept. As indicated by meta-analysis and systematic reviews, anti-CCP is not predictive for the response to anti-TNFs. ${ }^{2-4}$ Additional studies are needed to evaluate whether abatacept could be a preferable treatment in anti-CCP-positive pts.

References:

[1] Taylor P, et al. Autoimmune Dis 2011;2011:815038.

[2] Lv Q, et al. PLoS ONE 2014:9:e89442.

[3] Isaacs JD, et al. Ann Rheum Dis 2013;72:329-36.

[4] Cuppen BV, et al. Rheumatology (Oxford) 2016;55:826-39.

[5] Gottenberg JE, et al. Ann Rheum Dis 2012;71:1815-19.

[6] Sokolove J, et al. Ann Rheum Dis 2015;74(Suppl 2):675.

[7] Huizinga TWJ, et al. Ann Rheum Dis 2015;74(Suppl 2):234-5.

Disclosure of Interest: A. den Broeder Grant/research support from: CZ, Menzis, ZonMw, Consultant for: Amgen, Boehringer Ingelheim, Speakers bureau: BristolMyers Squibb, Pfizer, T. Kerstens: None declared, J. Fransen Grant/research support from: Bristol-Myers Squibb, C. van den Ende: None declared, L. Tweehuysen: None declared, R. Postema Employee of: Bristol-Myers Squibb, E. Alemao Shareholder of: Bristol-Myers Squibb, Employee of: Bristol-Myers Squibb,

F. van den Hoogen Consultant for: Celltrion, Sandoz, Mundipharma and Biogen, Speakers bureau: Celltrion, Sandoz, Janssen, Egis

DOI: 10.1136/annrheumdis-2017-eular.2477 